\title{
An Appreciative Inquiry Approach to Understanding What Attracts and Retains Early-Career Therapists to Work in Community Organizations
}

Karina M. Dancza

Singapore Institute of Technology, karina.dancza@singaporetech.edu.sg

Yik Ming Choi

Singapore Institute of Technology, jasonchoi@ymail.com

Kaisah Amalia

Singapore Institute of Technology and Woodlands Health Campus, 1602611@SIT.Singaporetech.edu.sg

Pei Shan Wong

Singapore Institute of Technology, 1602062@sit.singaporetech.edu.sg

Jia Hao Hu

Singapore Institute of Technology-Trinity College Dublin, 1602685@sit.singaporetech.edu.sg

See next page for additional authors

Follow this and additional works at: https://nsuworks.nova.edu/ijahsp

Part of the Occupational Therapy Commons, Physical Therapy Commons, and the Physiotherapy Commons

\section{Recommended Citation}

Dancza KM, Choi Y, Amalia K, Wong P, Hu J, Yap L. An Appreciative Inquiry Approach to Understanding What Attracts and Retains Early-Career Therapists to Work in Community Organizations. The Internet Journal of Allied Health Sciences and Practice. 2022 Jan 03;20(1), Article 7.

This Manuscript is brought to you for free and open access by the College of Health Care Sciences at NSUWorks. It has been accepted for inclusion in Internet Journal of Allied Health Sciences and Practice by an authorized editor of NSUWorks. For more information, please contact nsuworks@nova.edu. 


\title{
An Appreciative Inquiry Approach to Understanding What Attracts and Retains Early-Career Therapists to Work in Community Organizations
}

\begin{abstract}
Purpose: Community health organizations are expanding in many countries to meet the health and care needs associated with an aging population, an increase in recognition of children with developmental and mental health needs, and escalating healthcare costs. Singapore is no exception. Community organizations, however, are facing challenges in recruiting and retaining early-career therapists to these positions due to competition from acute services and a declining foreign talent pool. At the same time, early-career therapists encounter challenges during the transition from student to professional. Community organizations, therefore, need to identify what makes their organization attractive to earlycareer therapists and what makes them stay. This study explored factors that attracted and retained early-career therapists in community organizations in Singapore from the perspectives of early-career therapists and supervisors. Methods: Appreciative Inquiry was adopted as a strengths-based approach to guide this qualitative study process. Focus groups were conducted with three groups of early-career therapists and supervisors, to explore perspectives of what attracted them to work in a community health organization and what factors retained them in their roles. Two phases of focus groups were held threeweeks apart, giving early-career therapists and supervisors the opportunity to consolidate and expand their ideas. Focus groups were audio-recorded, transcribed, and thematically analyzed. Results: This study highlighted three key factors which attracted and retained early-career therapists to the Singapore community health organizations: (1) a culture that values learning and professional development; (2) structured supervision that is timely and individualized; and (3) a system that supports job satisfaction and promotes staff wellbeing. Conclusions: This study highlighted that community organizations could enhance their attraction and retention of early-career therapists through a focus on opportunities for earlycareer therapists' formal and informal learning and professional development opportunities. Having a clear supervision framework and continuing development for supervisors is another key attraction for earlycareer therapists. Finally, time and opportunities to develop meaningful relationships with colleagues and clients enhances job satisfaction and wellbeing of early-career therapists. Making explicit the offer in these three areas could enhance applications and the retention of early-career therapists to these services.
\end{abstract}

\section{Author Bio(s)}

Karina M Dancza, BAppSc(OT), MA(SEN), PGCLT(HE), Ph.D., is an Assistant Professor in Health and Social Sciences at Singapore Institute of Technology. ORCID ID http://orcid.org/0000-0003-3291-4467

Yik Ming Choi, BSc (Hons) PT, MMT, DClinPhysio, is a practicing physical therapist and an associate faculty in Health and Social Sciences at Singapore Institute of Technology.

Kaisah Amalia, BSc (Hons) OT, is a graduate in Bachelor of Science with Honours in Occupational Therapy from Singapore Institute of Technology and practicing occupational therapist at Woodlands Health Campus, Singapore.

Pei Shan Wong, BSc (Hons) OT, is a graduate in Bachelor of Science with Honours in Occupational Therapy from Singapore Institute of Technology.

Jia Hao Hu, BSc (Hons) PT, is a graduate in Bachelor of Science with Honours in Physiotherapy from Singapore Institute of Technology-Trinity College Dublin.

Li Wen Yap, BSc (Hons) OT, is a Principal Occupational Therapist in AWWA Ltd, Singapore. 


\section{Acknowledgements}

The authors acknowledge with gratitude Mr. Justyn Tan and Ms. Fanyi Foo from AWWA Ltd for their contributions as site coordinators and the therapists from AWWA Ltd who willingly shared and participated in this study.

\section{Authors}

Karina M. Dancza, Yik Ming Choi, Kaisah Amalia, Pei Shan Wong, Jia Hao Hu, and Li Wen Yap 


\title{
IIIAHSP \\ The Internet Journal of Allied Health Sciences and Practice \\ Dedicated to allied health professional practice and education
}

Vol. 20 No. 1 ISSN 1540-580X

\section{An Appreciative Inquiry Approach to Understanding What Attracts and Retains Early-Career Therapists to Work in Community Organizations}

\author{
Karina M. Dancza1 \\ Yik Ming Choi ${ }^{1}$ \\ Kaisah Amalia² \\ Pei Shan Wong ${ }^{1}$ \\ Jia Hao Hu${ }^{3}$ \\ Li Wen Yap ${ }^{4}$
}

1. Singapore Institute of Technology

2. Singapore Institute of Technology and Woodlands Health Campus

3. Singapore Institute of Technology - Trinity College Dublin

4. Asian Women's Welfare Association Ltd.

Singapore

\begin{abstract}
Purpose: Community health organizations are expanding in many countries to meet the health and care needs associated with an aging population, an increase in recognition of children with developmental and mental health needs, and escalating healthcare costs. Singapore is no exception. Community organizations, however, are facing challenges in recruiting and retaining early-career therapists to these positions due to competition from acute services and a declining foreign talent pool. At the same time, earlycareer therapists encounter challenges during the transition from student to professional. Community organizations, therefore, need to identify what makes their organization attractive to early-career therapists and what makes them stay. This study explored factors that attracted and retained early-career therapists in community organizations in Singapore from the perspectives of earlycareer therapists and supervisors. Methods: Appreciative Inquiry was adopted as a strengths-based approach to guide this qualitative study process. Focus groups were conducted with three groups of early-career therapists and supervisors, to explore perspectives of what attracted them to work in a community health organization and what factors retained them in their roles. Two phases of focus groups were held three-weeks apart, giving early-career therapists and supervisors the opportunity to consolidate and expand their ideas. Focus groups were audio-recorded, transcribed, and thematically analyzed. Results: This study highlighted three key factors which attracted and retained early-career therapists to the Singapore community health organizations: (1) a culture that values learning and professional development; (2) structured supervision that is timely and individualized; and (3) a system that supports job satisfaction and promotes staff wellbeing. Conclusions: This study highlighted that community organizations could enhance their attraction and retention of early-career therapists through a focus on opportunities for earlycareer therapists' formal and informal learning and professional development opportunities. Having a clear supervision framework and continuing development for supervisors is another key attraction for early-career therapists. Finally, time and opportunities to develop meaningful relationships with colleagues and clients enhances job satisfaction and wellbeing of early-career therapists. Making explicit the offer in these three areas could enhance applications and the retention of early-career therapists to these services.
\end{abstract}

Keywords: Allied health professionals, occupational therapists, physiotherapists, early-career therapists, new graduates, supervisors, attraction and retention, employee retention, recruitment, transition to practice, supervision, support, appreciative inquiry 


\section{INTRODUCTION}

Community and rural health services are rapidly expanding in response to an increasing demand associated with aging populations, an increased recognition of children with developmental and mental health needs, and rising healthcare costs, amongst others. ${ }^{1-3}$ Singapore is no exception, with community health services growing in recent years to sustainably meet healthcare needs ${ }^{4}$ Allied health professionals such as occupational therapists and physiotherapists are critical to the delivery of these expanding community health services and are recognized in the $3^{\text {rd }}$ Enabling Masterplan, a national roadmap for the disability sector from 2017 to $2021 .{ }^{5}$ Attracting and retaining this skilled workforce into community organizations, is thus seen as vital.

While there has been a greater emphasis on recruiting more allied health professionals into rural and community settings, these organizations are facing challenges due to competition from well-structured acute health care institutions in the cities and a declining foreign talent pool.1,6 In a city-state such as Singapore, services are not geographically distant, but community health organizations are experiencing rapid growth. While this situation is perhaps unique to Singapore, challenges faced appear to share some similarities with reports of rural and remote recruitment. For example, the community support structures are needing to quickly evolve and adapt, and there is significant competition from well-established acute services for personnel. In addition, many experienced therapists have settled in their existing workplaces in the acute services. Hence it is unlikely for them to move into new community positions, where there is a perceived difference in working conditions, further limiting the potential pool of applicants..$^{6,7}$ Moreover, recruitment is a costly process as resources are allocated to develop the skills of new employees. ${ }^{8}$ As such, it is important to understand what attracts a potentially greater pool of early-career therapists to join community organizations and what are the critical features which retain them in these roles.

Early-career therapists face many challenges during the transition from student to professional, such as a mismatch between expectations and reality of practice, and adapting to the evolving healthcare landscape. ${ }^{9}$ Early-career therapists are generally more sensitive to the quality of their working environment, and often require more support and guidance; something which may not be as available in rapidly expanding services. ${ }^{9}$ Another challenge includes potentially less access to professional development opportunities, when opportunities for professional development are reported to one of the key factors in the attraction and retention of healthcare professionals. ${ }^{10-12}$ For example, several studies have strongly supported the importance of ensuring availability of relevant professional development activities, and found that organizations who provided these opportunities and fostered a culture of learning, contributed to the retention of therapists. ${ }^{13}$

Supervision also plays an important role in assisting the transition of early-career therapists into practice, with good quality supervision contributing to staff retention. ${ }^{9}$ Guidelines for supervision have been developed by allied health professional bodies in different countries such as the USA, Australia, and Singapore..$^{14,15}$ Integral elements of supervision for early-career therapists include incorporating observation, feedback, and demonstration of practical components with clients. ${ }^{16}$ Reflective practice has also been recognized as an essential component of supervision that assists early-career therapists in identifying their learning goals. ${ }^{10}$

The frequency and duration of supervision have also been recommended by allied health professional bodies in different regions. In Singapore, early-career therapists undergo mandated supervision for a year, adhering to a set of supervised practice guidelines. Supervision intensity decreases over the year, from four hours a week, to four hours a fortnight, to eventually a month. ${ }^{15}$ In other countries like Australia, the timeframe for completion of supervision was not specified, and in the United Kingdom, no fixed recommendations have been provided on supervision. ${ }^{17,18}$ As supervision guidelines and approaches vary in different countries, it is important to consider the needs of early-career therapists within their context of practice.

Other than supervision, support for early-career therapists also includes team bonding and the ability to rely on colleagues when experiencing a heavy workload and challenging clients. ${ }^{7}$ The presence of support groups and peer support also fulfills the need of early-career therapists for shared reflection and feedback outside of formal supervision. ${ }^{16,19} \mathrm{In}$ contrast, absence of these supports reportedly impacts on wellbeing, which influences the retention rate of staff due to high burnout and low job satisfaction. ${ }^{20,21}$ Team bonding and peer support, coupled with supervision, are considered essential for early-career therapists to integrate successfully into the workplace and manage both challenging and rewarding experiences at work. ${ }^{22}$

Studies exploring the perspectives of both early-career therapists and supervisors on the factors attract and retain early-career therapists are generally limited. Three studies have explored recruitment and retention of the rural workforce from the perspectives of managers, senior therapists, and policymakers. ${ }^{16,23,24}$ Some recommendations proposed by Veitch et al. included scholarships for early-career therapists, financial incentives, and flexible work arrangements. ${ }^{24}$ However, these papers explored the challenges faced by rural regions and an acute hospital, which presented a different scope from this study. Thus, this study aimed to explore 
factors that attracted and retained early-career therapists in a community organization in Singapore from the perspectives of the early-career therapists and supervisors.

\section{METHODS}

The Consolidated Criteria for Reporting Qualitative Research (COREQ) frames this section and will elaborate on (1) research team and reflexivity, (2) study design, and (3) analysis. ${ }^{25}$

\section{Research Team and Reflexivity \\ Personal Characteristics and Relationship with Participants}

The study was conducted as part of undergraduate students' final year honours projects under the supervision of academic and community organization supervisors (co-investigators), collectively known as the "research team." Students were from occupational therapy (2 students) and physiotherapy (1 student) undergraduate programmes. The academic supervisors were one faculty from occupational therapy and one faculty from physiotherapy, both of whom have worked clinically and with experience of staff recruitment. The co-investigators were two occupational therapists and one physiotherapist who had been with the organization for over 2 years and had experiences of their own transitions as an early-career therapist. The community organization coinvestigators were known to the participants as colleagues within the same organization but were not in a direct managerial relationship.

The study was conceived by the community organization as they projected a growing need for allied health staff and wanted to tailor their offer to attract and retain quality candidates to their setting. The study was designed collaboratively with the community organization co-investigators, academic faculty, and students. The students led the focus group discussions with oversight from the co-investigators and academic faculty. The students undertook the initial data analysis, and the co-investigators and academic faculty were involved in refining the results and interpretations.

\section{Study Design \\ Theoretical Framework: Appreciative Inquiry}

This was a qualitative study conducted based on a constructionist, appreciative inquiry (Al) approach. From a constructionism perspective, an understanding of the important features and critical aspects of attracting and retaining early-career therapists to community settings was determined from analysis of the different perspectives of the early-career therapists and supervisors. ${ }^{26} \mathrm{As}$ the research team also had experiences related to the phenomenon, it enabled a rich understanding of the experiences to be reported. While some may consider closeness could compromise the research through bias, others (including this research team) hold the view that this closeness can promote an understanding of the participants' experiences, which enhances the trustworthiness and authenticity of the research. ${ }^{27}$

$\mathrm{Al}$ is a strength-based approach that emphasizes the positive aspects instead of focusing on the problems. ${ }^{28} \mathrm{Al}$ is reported to have a significant potential for transformation by building on what works well and using that to initiate change. It is characterised by four phases known as the 4D cycle (Figure 1), which consist of discovery, dream, design and deliver. ${ }^{28}$ The discovery phase focuses on appreciating what is working well, while the dream phase explores what might be and participants are encouraged to imagine beyond boundaries. The design phase focuses on practical steps needed to move closer to the ideal situation. Lastly, the deliver phase is about the commitment to change and how to sustain this envisioned future. ${ }^{28}$

\section{Ethical Considerations}

Ethical approval was granted by the Institutional Review Board of Singapore Institute of Technology and informed consent was obtained prior to the start of data collection.

\section{Participant Selection and Recruitment}

Consistent with qualitative research, participants were early-career therapists and supervisors who were purposively recruited from a community health organization in Singapore. ${ }^{29}$ Early-career therapists included the occupational therapists and physiotherapists who were undergoing or had completed their one-year mandated supervision in the community organization. Only therapists who had joined the community organization between 2014 and 2019 were included as they had between one to five years of experience, and hence considered "early-career." Supervisors were invited to participate if they had provided mandatory supervision for at least one therapist in the organization. This deliberate selection strategy enabled information-rich participants to be included to maximize understanding of the phenomenon under investigation..$^{29}$ 
All potential participants in the organization were approached via email invitation from a co-investigator (early-career therapists approximately 20 occupational therapists and 30 physiotherapists: supervisors - approximately 44 occupational therapists and 7 physiotherapists). A total of 20 participants agreed and were available to participate, including 12 early-career therapists and 8 supervisors (please see Table 3). Six focus group discussions were held ( 2 for the supervisors and 4 for the early-career therapists). Hennik and colleagues found that two focus groups for each group of participants were sufficient to understand the phenomena of interest thoroughly. ${ }^{30}$

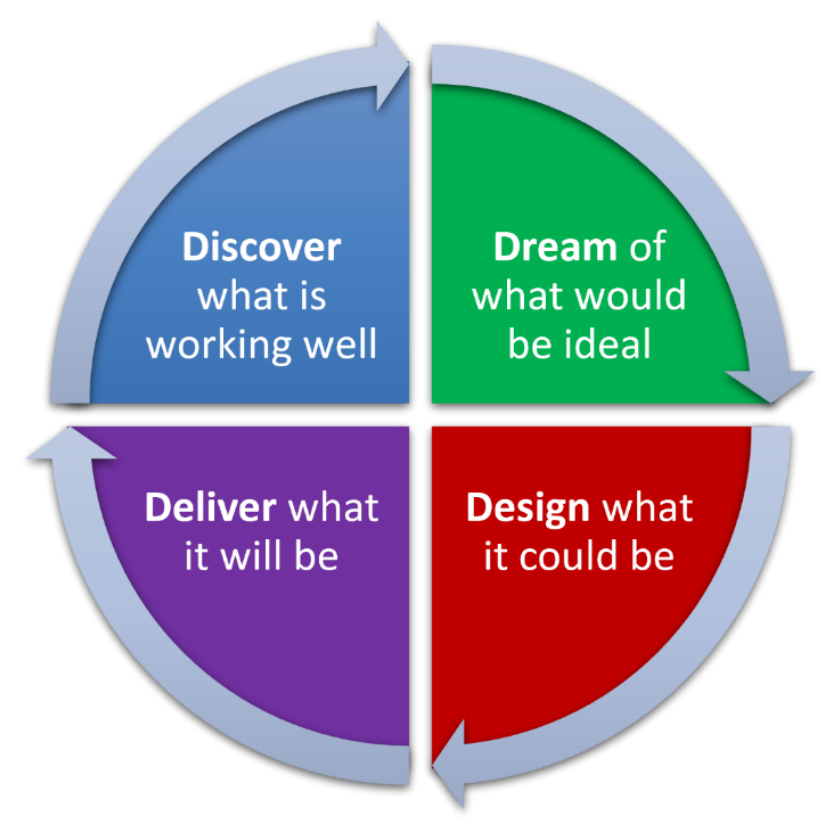

Figure 1: Appreciative Inquiry 4D cycle (adapted from Cooperrider, Whitney and Stavros) ${ }^{28}$

\section{Setting}

All focus groups were held at the community organization headquarters in a private meeting room. Two student researchers facilitated each focus group, with the other student researcher, co-investigators, and academic faculty present to collect fieldnotes relating to key points and reactions of the participants to aid in transcription and interpretation of the data. At times, the coinvestigators or academic faculty also asked participants clarifying or probing questions to support the student facilitators to add richness to the data.

\section{Data Collection}

All participants were divided into two groups, namely early-career therapists and supervisors. The early-career therapists were further divided into two groups to facilitate in-depth discussions and help with scheduling of the focus groups. Each group underwent two phases of audio-recorded focus group discussions, lasting between 90-120 minutes each. Open-ended questions and prompts were developed based on the four phases of Al cycle. Phase One focus groups addressed questions relating to the "Discover" and "Dream" stages (Table 1) while Phase Two targeted questions based on the "Design" and "Deliver" stages (Table 2). ${ }^{28}$ Questions included the exploration of positive experiences of working in the community organization, envisioning an ideal experience as a community therapist, creation of this ideal experience and resources required, and sustaining the ideal experience.

There was a three-week interval between the two phases to allow for verbatim transcription of audio recordings as well as preliminary data analysis in preparation for Phase Two. ${ }^{28}$ This preliminary analysis involved all members of the research team reviewing the data and engaging in reflective discussions about the key information. Four themes were developed to capture the main elements of the data: (1) professional development roadmap, (2) guidance for supervision and mentoring, (3) ecosystem for support and growth and (4) culture of social wellbeing. These four themes were presented and explored with all participants in the design and deliver phase of the Al cycle in Phase Two, allowing for member checking of the initial findings. ${ }^{25}$ As this study used 
constructionism perspectives, this preliminary analysis enabled information to be shared among all three groups of participants to gather a variety of perspectives for the final analysis. ${ }^{26}$

Table 1: Phase one focus group questions relating to the "Discover" and "Dream" stages

\begin{tabular}{|c|c|}
\hline Early-Career Therapists & Supervisors \\
\hline $\begin{array}{l}\text { What attracted you to join this organization? } \\
\text { What are some of your positive experiences while } \\
\text { working in this setting? }\end{array}$ & $\begin{array}{l}\text { Why do you think early-career therapists are attracted to your } \\
\text { organization? }\end{array}$ \\
\hline $\begin{array}{l}\text { What are the positive experiences that are currently } \\
\text { making you stay within the organization? }\end{array}$ & $\begin{array}{l}\text { What has been implemented by your organization that has } \\
\text { successfully: } \\
\text { - attracted early-career therapists to the setting? } \\
\text { - retained early-career therapists in the setting? }\end{array}$ \\
\hline $\begin{array}{l}\text { What could have made your experiences even better } \\
\text { - in terms of your development and growth as a } \\
\text { therapist? } \\
\text { - in terms of learning and working environment? }\end{array}$ & $\begin{array}{l}\text { Are there any other ways in which your organization created } \\
\text { positive experiences that contributed to attracting or retaining } \\
\text { early-career therapists? }\end{array}$ \\
\hline $\begin{array}{l}\text { What would be an ideal type of experience that would } \\
\text { encourage you to stay? }\end{array}$ & $\begin{array}{l}\text { What do you think can be done to improve these existing } \\
\text { programs to further attract and retain more early-career } \\
\text { therapists? }\end{array}$ \\
\hline
\end{tabular}

Table 2 Phase two focus group questions relating to the "Design" and "Deliver" stages

Early-Career Therapists
How do you think the positive experiences could be
enhanced in this organization? What could be
implemented?

What resources are needed for this to occur?

What further ideas could be implemented by the management/organization to encourage you to stay in the community organization?

What do you think can be achieved in the next one week / one month / six months / one year?

Which of the suggestions can be achieved in the next one month / six months / one year?

How do you think the suggestions identified above can be sustained?

\section{Supervisors}

What other programs/services do you think can be

implemented to:

- Attract more early-career therapists?

- Retain more early-career therapists?

What do you think is an ideal experience for the early-career therapists in the community organization?

- In terms of their development and growth as a therapist

- In terms of their learning and working environment

What resources are needed for these programs to happen / to create the ideal experience for the early-career therapists?

From a leadership perspective, what can you do to make them stay?

As a supervisor in the organization, what can you do to create the ideal experience for the early-career therapists?

\section{Data Analysis}

All focus groups were transcribed and analyzed through thematic analysis guided by Braun and Clarke. ${ }^{31}$ Firstly, three researchers worked separately to read through all the six individual transcripts before setting preliminary codes to sort the data. Subsequently, codes were reviewed and refined collectively reaching a consensus with the entire research team, thereby enhancing coding reliability. QSR International's NVivo 12 Plus Software was used to collate and organized the codes into broader themes. ${ }^{32}$ Themes were then re-examined by the research team to determine if they were relevant and accurate to the coded references and entire data-set. The themes were frequently refined to enhance the specificity of each theme and ensure accuracy to the original data. ${ }^{31}$ RESULTS

A total of 20 participants took part in the focus groups. The demographics of the participants are shown in Table 3. Four participants from the early-career therapist groups dropped out of the second focus group session. This comprised of two female occupational 
therapists and one male physiotherapist from one early-career therapists focus group, and one female occupational therapist from the other early-career therapists focus group. The reason given for their absence was due to work commitments and scheduling conflicts, and all agreed for their data from the first focus group to be retained and included in data analysis.

Table 3: Demographics of the participants

\begin{tabular}{lll} 
& Early-career therapists $(\mathbf{n}=\mathbf{1 2})$ & Supervisors $(\mathbf{n}=\mathbf{8})$ \\
\hline Discipline & & 5 \\
\hline Occupational Therapists & 7 & 3 \\
\hline Physiotherapists & 5 & \\
\hline Gender & & 5 \\
\hline Female & 7 & 3 \\
\hline$\quad$ Male & 5 & 0 \\
\hline Dropout (second session) & 4 &
\end{tabular}

Three key themes were developed from the data: (1) a culture that values learning and professional development; (2) structured supervision that is timely and individualized; and (3) a system that supports job satisfaction and promotes staff wellbeing. Each theme is elaborated below, focusing on what participants from all groups felt was working well and what might be further enhanced within the organization, consistent with the constructionist, Al perspectives of the research.

\section{A Culture that Values Learning and Professional Development}

Early-career therapists and supervisors described how they appreciated the culture within the organization that valued learning and professional development. Specifically, they spoke about how the organization explicitly focused on their career progression and made plans with them for a personal career pathway.

"You start talking about career progression from [the moment] you joined as a junior therapist. So, it's good that at least they explore with us the moment we step into the company." (Early-career therapist 19; Focus Group Phase One)

"...we also do have clinical heads who look into [your] career right from the beginning as well. Like what you want to do, where your interests are, where [you see] yourself in five-years down the line." (Supervisor 13; Focus Group Phase One)

Early-career therapists discussed how they have been given many opportunities to develop themselves through attending courses and sharing their knowledge with others through presenting at training events.

"[The organization] gives the junior therapists a lot of chances to explore a lot of things. Like for example they send us to courses they feel are relevant to us and will be beneficial for us. Also, they give us a lot of chances to do public talks, do volunteer training or therapy assistant training, these kinds of things. So, they give us a lot of chance to not only train us clinically, [but also] in other aspects like how we talk to the public, and how we can conduct a small training [event]." (Early-career therapist 18; Focus Group Phase One)

"For learning wise, I think it is better in the community because you can learn from the different therapists like ST [speech therapy], PT [physiotherapy] and OT [occupational therapy]. You can see what others are doing." (Early-career therapist 3; Focus Group Phase One)

Opportunities to learn from other professionals and explore different service areas within the organization were also expressed as reasons for staying in the job. While there were some opportunities to do this within the organization, therapists shared that having these attachments across other organizations in both the acute and community organizations would be useful to build on their clinical exposure within the industry.

"Another factor that makes me want to stay [with the organization] is because you can rotate to a lot of different settings. So, now I am based at the nursing home and then I will want to try day rehabilitation or even like home therapy... There's a lot of opportunities here." (Early-career therapist 2; Focus Group Phase One) 
"So, I think (the organization) could probably explore the option of offering physiotherapists to the acute hospitals. So, I think junior physiotherapists kind of require some kind of clinical experience in the acute side before actually getting out in the community so maybe that's something that (the organization) should look into." (Early-career therapist 15; Focus Group Phase One)

A focus on learning, and opportunities for professional development were highly valued by early-career therapists and contributed to their willingness to continue their career within the organization. However, as novice professionals, they also expressed how they would appreciate further structure and guidance on how to plan their learning and career progression. Proposed ideas for improving professional development systems included labelling learning opportunities according to the expected level of experience required to gain the most from it, and further clarifying how to move from a junior to senior therapy position.

"When you just start, you don't know what you don't know, what you have or what is there to explore. Maybe [having a] topic bank, [with] suggestions according to your level [would be helpful]. For example, when reaching the second or third year and moving into a leadership position, what courses would be suitable for you and how could you progress from there?" (Early-career therapist 3; Focus Group Phase Two)

"[lt would be helpful to have] more defined measures on how we want [early-career therapists to advance]. Then we could look at career progression for our therapists from junior to senior, that would be a better way that we could help them foresee their future in [the organization]" (Supervisor 8; Focus Group Phase One)

\section{Structured Supervision that is Timely and Individualized}

Early-career therapists appreciated consistency in supervision with the clearly defined roles of having a supervisor to support their clinical issues and a reporting officer who was their line manager.

"In this organization, each discipline has a therapy head. I thought that was good, so you know who to turn to whenever you have [clinical] issues. For operational issues, it is your RO [reporting officer] ... There is no overlap of roles among the management." (Early-career therapist 5; Focus Group Phase One)

Supervision was valued by early-career therapists, with some expressing how they would like their supervision extended longer than the mandated period, while others stating that the amount provided was appropriate.

"Usually after completing our [mandated period of] supervision, we are being let go of. What I want to see is the same consistent mentorship even after finishing our [mandated period of] supervision, at least until we are more confident, maybe for two to three more years." (Early-career therapist 5; Focus Group Phase One)

"I feel like they have provided me with sufficient hours of supervision. They don't really overload you with too much supervision as well." (Early-career therapist 19; Focus Group Phase One)

Supervisors considered it important to have dedicated time to fulfil this role so they could provide quality supervision. Supervisors, however, expressed how allocating the required time for supervision was already challenging with high workloads and they did not know how an additional supervision load (if the mandated supervision period were extended) would be practical.

"We have a client ratio that is quite high... So, when [it] is considered like that, I need to fulfil my clinical responsibilities as well as my mentorship, as well as my senior responsibilities, which is not practically so good on the supervisors. But it will be good to have adequate time for [supervisors] to [fulfill] these types of responsibilities." (Supervisor 6: Focus Group Phase One)

Participants acknowledged that different ways of providing supervision could be a way of addressing the wish of some early-career therapists for an extension to the period of intensive supervision within existing resources. For example, suggestions included using a multiple mentoring model where a few supervisors shared responsibility for a group of supervisees, or extending the peer support system which was already in place in some areas of the organization to the entire setting.

"Having a few supervisors who are okay to work together to support that same staff [early-career therapists], I think that would be easier [as] the supervisors can share the load... I think that it would [be] easier to meet the needs of the supervisees and [allow] the supervisor [to] cope with it as well." (Supervisor 9: Focus Group Phase Two) 
"[Having] a buddy [scheme] with maybe another junior therapist [would be helpful]. We will be more comfortable in communicating with them rather than to a senior physiotherapist" (Early-career therapist 16; Focus Group Phase Two)

Within existing structures, supervisors used a range of tools and strategies to support early-career therapists during supervision, such as reflective templates to guide learning.

"... we actually have this staff reflection form that we go through with the [early-career therapists] to think about what kind of support they want... the form does help to guide their thoughts a little bit to work on other areas that they want to improve.... [It also makes explicit] what are the expectations that they have of their supervisors [and what] they want us to support them in." (Supervisor 9; Focus Group Phase Two)

While the supervision strategies used were described as helpful, both supervisors and early-career therapists suggested that further training or peer mentoring for supervisors would enhance current practices.

"Maybe they can have a training for themselves also, like the supervisors?" (Early-career therapist 19; Focus Group Phase One)

"[Having] a framework for how you should supervise the mentee [would be helpful]. Like let's say you let the mentee do whatever with the client [and] after the session [the supervisor] asks what went well and what could be improved or what do you think you did good or what not, so there's a lot of reflection involved which I think isn't really happening." (Earlycareer therapist 15: Focus Group Phase Two)

"For the supervisors, we also need support. Because we have been supervising for so long, and we think that we are expert in this area, but we may not know what's our blind spots, so we need that guidance as well, from another person." (Supervisor 13; Focus Group Phase Two)

\section{A System that Supports Job Satisfaction and Promotes Staff Wellbeing}

In the community organization, participants shared how the service structures and nature of the job meant they were able to develop meaningful relationships with their clients as they could consistently work with people over time and in their usual contexts. This was contrasted with faster paced acute settings. Early-career therapists valued the time they were able to spend with their clients and the progress they shared, as this gave them a sense of job satisfaction and reason to stay in their role.

"We actually have more time to spend with our patients if we are in a community setting rather than acute setting... in the community, we have a lot of time together with them to do the rehabilitation so you can really see the progression and I think there's a sense of satisfaction... to actually see them improve from day to day." (Early-career therapist 18; Focus Group Phase One)

"The fact that I get to see them every week...makes me want to stay... and you know ...looking at their progress" (Earlycareer therapist 2: Focus Group Phase One)

Early-career therapists described how the supportive nature of the organization and recognition of the importance of work-life balance were important to their decision to join and remain in their job. Supervisors expressed how time for life outside of work and meeting family commitments were recognized as vital for staff wellbeing.

"...the work - life balance is very stable so it's like only 40 hours per week. So, it is not like in acute setting, where we don't get that type of a balance because we can be on-call anytime or you may work 44 hours per week so that is one reason why I chose [to work in the community organization]." (Early-career therapist 16; Focus Group Phase One)

"If the therapist needs a time for their family, [the organization] also appreciates the importance of family and work. [The organization] can provide therapists with the necessary support so that they can [take care of] their family before they can come back to the work for instance." (Supervisor 13; Focus Group Phase One)

In addition, early-career therapists also spoke about how the organization acknowledged the importance of social networks. Support from colleagues in managing the daily stress associated with being a healthcare professional was seen as vital and encouraged through initiatives such as team bonding activities and informal lunches together. 
"I think social support is important not just in community but even in acute services because healthcare has a high burnout rate, so you definitely need that social support. Usually what makes work easier is your colleagues. If you have a strong team, a good team, then whatever difficulties are faced at work, it is just something to overcome." (Early-career therapist 2; Focus Group Phase One)

"There is an initiative where we actually encourage team gatherings, we actually encourage staff bonding, like even just lunch together... to actually encourage the team to work more cohesively and to not talk about just work during working hours, we can talk about other stuff." (Supervisor 9; Focus Group Phase One)

\title{
DISCUSSION
}

The aim of this study was to explore aspects within community organizations that attracted and retained early-career therapists to these roles. Engaging in a strength-based, appreciative inquiry approach to capture positive features meant that aspects of good practice were identified, and areas for further enhancement suggested. ${ }^{28}$ The results of this study highlighted three key factors which attracted and retained early-career therapists to community organizations (Figure 2): (1) a culture that values learning and professional development; (2) structured supervision that is timely and individualized; and (3) a system that supports job satisfaction and promotes staff wellbeing.

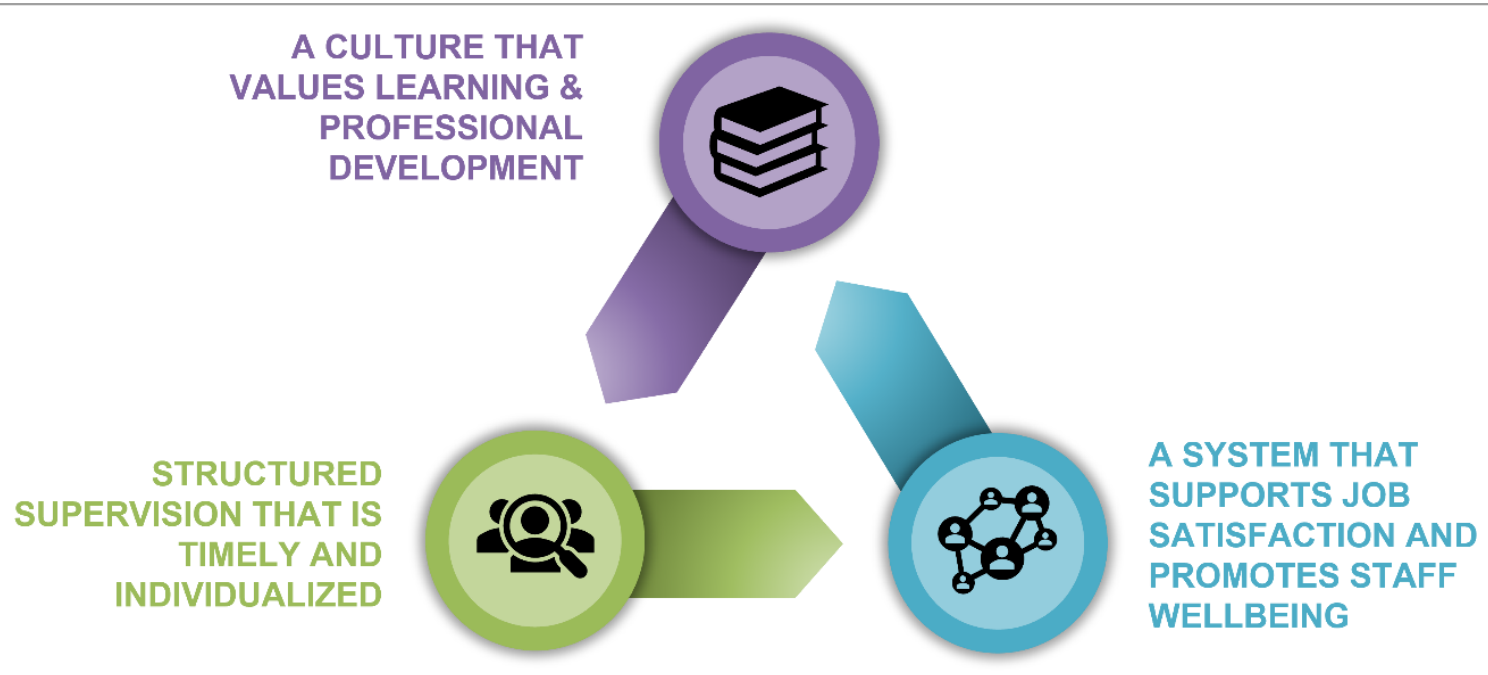

Figure 2: Factors that attract and retain early-career therapists to work in community organizations

\begin{abstract}
A Culture that Values Learning and Professional Development
Opportunities for learning and professional development were seen by both supervisors and early-career therapists as an important factor in their choice to join and stay with the community organization. Participants described how they valued the range of learning opportunities currently offered within their organization, from course attendance and studying for formal qualifications, to learning from colleagues and opportunities to shadow others in different services. This created an ecosystem of learning where participants could design their professional development activities to suit their individual needs. Learning opportunities were especially important during the transition from student to graduate, when they were experiencing a steep development of their knowledge and skills. ${ }^{9}$ This reflects existing literature that has linked access to learning and professional development opportunities with job satisfaction and is a key predictor of staff retention. ${ }^{11,12,33}$
\end{abstract}

Although provision of ample learning opportunities was important, early-career therapists in this study also highlighted the need for guidance to make the most of these opportunities. The early-career therapists recognized that their limited depth and breadth of knowledge in the field often made it difficult to recognize their learning needs and prioritize where they should further develop. Guidance from supervisors and others in the organization was valued, although this was also an area with further development potential. Suggestions included having a clear career progression pathway and to label learning opportunities according to different levels of experience. This reflects the structure guidance offered by the Royal College of Occupational Therapists (RCOT) in the 
United Kingdom, in the Career Development Framework: Guiding Principles for Occupational Therapy. ${ }^{34}$ This framework presents nine career levels and four pillars underpinning practice (Professional Practice; Facilitation of Learning; Leadership; Evidence, Research and Development). Therapists can map their current position and plan their career progression. Having learning opportunities labeled with the level and pillar of practice potentially could assist early-career therapists' selection of relevant learning opportunities.

\section{Structured Supervision that is Timely and Individualized}

Participants in this study cited supervision as one of the crucial factors that attracted and retained early-career therapists to the community organization. This is consistent with other studies reporting that access to appropriate supervision is vital in recruitment and retention. ${ }^{35}$ Participants also appreciated the support given by the supervisors, especially when it comes to managing complex cases and establishing themselves in their professional role. According to Snowdon et al, supervision is effective in supporting the professional role of allied health professionals. ${ }^{14}$ Supervision was also reported to assist the early-career therapists in the transition to practice and played an important role in their personal and professional growth. This is a similar finding to Roots and Li, where they suggested the availability of professional and peer support were vital in attracting and retaining early-career therapists in rural services. ${ }^{12}$

Early-career therapists consistently described how important it was to have a clear structure for supervision to help them understand what was expected of them in this new work environment. Strategies described concur with Moores and Fitzgerald's findings and included using a clinical learning framework, protocols, and checklists. ${ }^{9}$ Early-career therapists and supervisors agreed that approaches to supervision should be tailored, using a mixture of observation, discussion, and hands-on practice.

The clear structure and different focal areas identified by participants as important could be enhanced with the adoption of relevant supervision models. For example, Proctor's three functions of supervision suggests three flexible and overlapping elements, including (1) Managerial / Administrative (e.g., caseload management, quality assurance); (2) Educational (e.g., professional and career development); and (3) Supportive (e.g., considering the emotional load associated with practice). ${ }^{36}$ Supervisors could use such models to tailor the learning approaches and make the outcomes of supervision clearer for early-career therapists (Figure 3 ).

\section{Supportive}

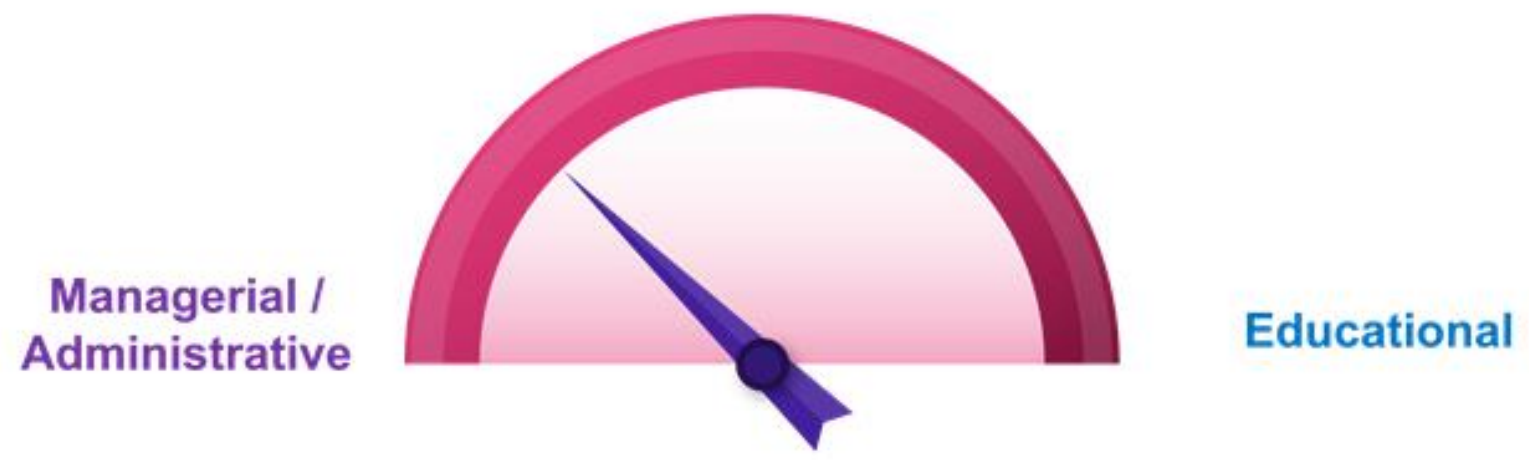

Figure 3: Balancing supervision activities (adapted from Proctor's three functions of supervision) ${ }^{36}$

Supervisors suggested more time for case discussions would be valuable, although additional time was not a main consideration for the early-career therapists. This could mean that they are satisfied with the time spent on supervision, but perhaps the activities during supervision could be further consolidated. In addition, multiple mentoring models of supervision was also suggested as a potential way to meet the demands of supervision, particularly beyond the mandated period. ${ }^{37}$

Another important element of effective supervision was timeliness. In line with findings from Moores and Fitzgerald, early-career therapists prioritized the immediacy of support, which could be peers or supervisors who were co-located. ${ }^{9}$ This is also consistent with other literature which suggests that working alone without easy access to supervisors and peers could lead to professional isolation, which was a strong predictor for leaving a job. ${ }^{11}$ At the time of this study, participants generally spoke of face-to-face supervision rather than technology platforms. While the role of tele-supervision, the remote delivery of clinical supervision through 
video conferencing software, was recently explored by Jordan and Shearer, the uptake of the use of technology has been accelerated with the recent global COVID-19 pandemic. ${ }^{38}$ American Occupational Therapy Association (AOTA) has suggested the possibility of using tele-supervision to support students and therapists working in isolated or rural areas, while the AHPC has also recently published guidelines for utilizing tele-supervision to continue support for early-career therapists in Singapore during the COVID pandemic. ${ }^{39,40}$ There is increasing potential for technology-based supervision systems to continue offering a platform for supervision in future, which could enhance access and timeliness for early-career therapists in community organizations.

The importance of training for the provision of supervision was also highlighted by early-career therapists and supervisors. This could be attributed to the complex and demanding nature of supervision hence the emphasis on training for supervisors. ${ }^{41}$ It is common for therapists to take on supervisory roles based on years of work experience, rather than interest, staff attributes or relevant training in supervising or mentoring staff. $15,18,42$ Peer mentoring for supervisors and formal education courses were suggested as possible strategies for supporting supervisors, but specifically what this would include warrants further exploration.

\section{A System that Supports Job Satisfaction and Promotes Staff Wellbeing}

Job satisfaction and emphasis from the organization on staff welfare was also a prominent factor in attracting and retaining the early-career therapists in this study. With the organization recognizing the importance of work-life balance, such as allowing flexibility in working arrangements to accommodate family life, early-career therapists and supervisors expressed how this influenced their decision to stay in the job. Consistent with Brough et al, an organization that understands the needs of the therapists that were not work-related, such as family needs, contributes to better work-life balance and this form of flexibility was crucial in recruitment and retention. 43

Early-career therapists and supervisors also highlighted supportive colleagues as a factor in their decision to stay in the community setting. This was supported by Gillham and Ristevski who described colleagues as significant in providing guidance and advice which was a central retention factor. ${ }^{35}$ Other reasons cited by therapists as to why they chose to work in rural areas was the desire to meet community needs. ${ }^{44}$ Similarly, in this study, early-career therapists valued the opportunity to work with clients over an extended period so they can form relationships and see meaningful changes. This was cited as another source of motivation to join and stay in the community organization.

\section{LIMITATIONS}

Appreciative Inquiry framed discussions from a strengths-based perspective, thus it may appear to be an idealistic form of gathering information. However, this process was selected as it was intended to identify what was working and what could be enhanced to attract and retain early-career therapists to the community organization. As such, the study wished to avoid an emphasis on problems which can perpetuate negativity and reduce potential for positive change. A positive approach was therefore adopted to act as a motivator for generating solutions. ${ }^{28}$

The study was carried out in one community organization in Singapore with a small number of participants. It is possible that the views shared may not be reflective of the broader community organizations within Singapore or elsewhere. As the results share similarities with the literature on recruitment and retention of early-career therapists in rural and remote settings, there is potential for the results to have some applicability in this broader context, and beyond Singapore. However, there are differences between community organizations in Singapore and the specific issues faced attracting and retaining personnel in rural and remote services, so these results may need to be interpreted with caution.

Unfortunately, four early-career therapists only participated in the first round of focus groups as they had scheduling conflicts at the time of the second round. The results may have been influenced by this dropout rate as their views were not represented in the discussions. However, as the themes between supervisors and early-career therapists showed significant overlap, it is possible that this did not have a major impact, although further research is recommended to confirm these findings.

\section{CONCLUSION}

The results of this study highlight the factors which attract and retain early-career therapists to community organizations and areas which would benefit from further development. Early-career therapists join and stay with a community organization when the culture of that organization values learning and professional development, has a structured, individualized, and timely approach to supervision, and systems which supports job satisfaction and promote staff wellbeing. The future goals are to enhance guidance for early-career therapists to make the most of available learning opportunities, offer additional support and education for supervisors potentially including supervision models and career development frameworks, and capitalizing on technology to enhance access to timely supervision. 


\section{REFERENCES}

1. Chin CWW, Phua KH. Long-term care policy: Singapore's experience. J Aging Soc Policy. 2016;28(2):113 -129. https://doi.org/https://doi.org/10.1080/08959420.2016.1145534. Published March 19, 2016. Accessed May 14, 2020.

2. Lim CG, Loh H, Renjan V, et al. Child community mental health services in Asia Pacific and Singapore's REACH model. Brain Sci. 2017;7(126):1-10. doi:10.3390/brainsci7100126.

3. William AH. Affordable Excellence: The Singapore Healthcare Story. Washington, DC: Brookings Institution Press; 2013.

4. Ministry of Health. Healthcare industry transformation map for a future-ready healthcare system. Press Release. https://www.mti.gov.sg/-/media/MTI/TMM/Essential-Domestic-Services/Healthcare/Healthcare-ITM---Press-Release.pdf. Published November 8, 2017. Accessed May 14, 2020.

5. The $3^{\text {rd }}$ Enabling Master Plan. Caring Nation, Inclusive Society. https://www.ncss.gov.sg/NCSS/media/NCSS-Documentsand-Forms/EM3-Final_Report_20161219.pdf. Published December 2016. Accessed May 14, 2020.

6. World Health Organization. Increasing Access to Health Workers in Remote and Rural Areas Through Improved Retention: Global Policy Recommendations. https://apps.who.int/iris/bitstream/handle/10665/44369/9789241564014_eng.pdf;jsessionid=245132D6618171C1AC8B0A1 BD332A888? sequence=1. Published 2010. Accessed May 14, 2020

7. Cosgrave $C$, Maple M, Hussain R. An explanation of turnover intention among early-career nursing and allied health professionals working in rural and remote Australia: Findings from a grounded theory study, Rural Remote Health. 2018;18(3):4511. doi:10.22605/RRH4511.

8. Gusdorf ML. Recruitment and Selection: Hiring the Right Person. https://cdn.ymaws.com/ise.org.uk/resource/resmgr/files/knowledge_reports/Recruitment_and_Selection_IM.pdf. Published 2008. Accessed May 14, 2020.

9. Moores A, Fitzgerald C. New graduate transition to practice: how can the literature inform support strategies? Aust Health Rev. 2017;41(3):308-312. doi:10.1071/AH15240.

10. Fitzgerald $C$, Moores $A$, Coleman $A$, et al. Supporting new graduate professional development: A clinical learning framework. Aust Occup Ther J. 2015:62(1):13-22. doi:10.1111/1440-1630.12165.

11. Gallego G, Dew A, Bulkeley $\mathrm{K}$, et al. Factors affecting retention of allied health professionals working with people with disability in rural New South Wales, Australia: Discrete choice experiment questionnaire development. Hum Resour Health. 2015;13(22):1-11. doi:10.1186/s12960-015-0013-7.

12. Roots RK, Li LC. Recruitment and retention of occupational therapists and physiotherapists in rural regions: A metasynthesis. BMC Health Serv Res. 2013;13(59):1-13. doi:10.1186/1472-6963-13-59.

13. Schoo A, Stagnitti $K$, Mercer $C$, et al. A conceptual model for recruitment and retention: Allied health workforce enhancement in Western Victoria, Australia. Rural Remote Health. 2005;5(4):1-18. doi:10.22605/RRH477.

14. Snowdon DA, Sargent M, Williams $C M$, et al. Effective clinical supervision of allied health professionals: A mixed methods study. BMC Health Serv Res. 2019;20(2):1-11. https://doi.org/10.1186/s12913-019-4873-8. Published December 31, 2019. Accessed May 21, 2020.

15. Allied Health Professions Council. Supervised Practice Guidelines for Occupational Therapists, Physiotherapists and Speech-Language Therapists (Conditional and Temporary Registration). https://www.healthprofessionals.gov.sg/docs/librariesprovider5/forms-and-downloads/010414_ahpc-supervised-practiceguidelines-(2014)_ver4.pdf?sfvrsn=6a31e77_0. Published 2014. Accessed May 14, 2020.

16. Cusick A, Mclntosh D, Santiago L. New graduate therapists in acute care hospitals: Priorities, problems and strategies for departmental action. Aust Occup Ther Jour. 2004;54(4):174-184. https://doi.org/10.1111/j.1440-1630.2004.00380.x. Published June 17, 2004. Accessed May 21, 2020.

17. Occupational Therapy Board of Australia. Code of Conduct: For Registered Practitioners. https://www.occupationaltherapyboard.gov.au/Codes-Guidelines/Code-of-conduct.aspx. Published March, 2014. Accessed June 22, 2020. 
18. College of Occupational Therapists. Supervision: Guidance for Occupational Therapists and Their Managers. United Kingdom, UK: Lavenham Press; 2015.

19. Morley M. Developing a preceptorship programme for newly qualified occupational therapists: Action research. Br J Occup Ther. 2007;70(8):330-338. doi:10.1177/030802260707000802.

20. Lau B, Skinner EH, Lo K, et al. Experiences of physical therapists working in the acute hospital setting: Systematic review. Phys Ther. 2016;96(9):1317-1332. doi:10.2522/ptj.20150261.

21. Scanlan JN, Still M. Job satisfaction, burnout and turnover intention in occupational therapists working in mental health. Aust Occup Ther J. 2013;60(5):310-318. doi:10.1111/1440-1630.12067.

22. Seah $\mathrm{CH}$, Mackenzie L, Gamble J. Transition of graduates of the master of occupational therapy to practice. Aust Occup Ther J. 2011;58(2):103-110. doi:10.1111/j.1440-1630.2010.00899.x.

23. Veitch C, Dew A, Bulkeley K, et al. Issues affecting therapist workforce and service delivery in the disability sector in rural and remote New South Wales, Australia: Perspectives of policymakers managers and senior therapists. Rural Remote Health. 2012;12(2):1903. doi:10.22605/RRH1903.

24. Moore T, Sutton KM, Maybery DJ. Rural mental health workforce difficulties: A management perspectives. Rural Remote Health. 2010;10(3):1-10. doi:10.22605/RRH1519.

25. Tong A, Sainsbury P, Craig J. Consolidated criteria for reporting qualitative research (COREQ): a 32 item checklist for interviews and focus groups. International Journal for Quality in Health Care. 2007;19(6):349-357. doi:10.1093/intqhc/mzm042.

26. Crotty M. The Foundations of Social Research: Meaning and Perspective in the Research Process. Los Angeles, USA: Sage; 1998.

27. Dwyer SC, Buckle JL. The Space Between: On Being an Insider-Outsider in Qualitative Research. International Journal of Qualitative Methods. March 2009:54-63. doi:10.1177/160940690900800105

28. Cooperrider DL, Whitney D, Stavros JM. Appreciative Inquiry Handbook: For Leaders of Change (2nd Ed.). Oakland, CA: Berrett-Koehler Publishers; 2008.

29. Braun V, Clarke V. Conceptual and design thinking for thematic analysis. Qualitative Psychology (in press). https://uwerepository.worktribe.com/output/7164974/conceptual-and-design-thinking-for-thematic-analysis

30. Hennink MM, Kaiser BN, Weber MB. What influences saturation? Estimating sample sizes in focus group research. Qualitative Health Research. 2019;29(10):1483-1496. doi:10.1177/1049732318821692

31. Braun V, Clarke V. Using thematic analysis in psychology. Qual Res in Psychol. 2006;3(2):77-101. doi:10.1191/1478088706qp063oa.

32. QSR International. NVIVO 12 Plus Software. https://www.qsrinternational.com/nvivo-qualitative-data-analysissoftware/home. Published 2018. Accessed 2019-2020.

33. Keane S, Smith T, Lincoln M, et al. Survey of the rural allied health workforce in New South Wales to inform recruitment and retention. Aust J Rural Health. 2011;19(1):1440-1584. doi:10.1111/j.1440-1584.2010.01175.x.

34. Royal College of Occupational Therapists. The Career Developmental Framework: Guiding Principles for Occupational Therapy. $2^{\text {nd }}$ Edition. London: Royal College of Occupational Therapists; 2021. https://www.rcot.co.uk/publications/careerdevelopment-framework

35. Gillham S, Ristevski E. Where do I go from here: We've got enough seniors? Aust J Rural Health. 2007;15(5):313-320. doi:10.1111/j.1440-1584.2007.00900.x.

36. Proctor B. Training for the supervision alliance attitude, skills and intention, In JR Cutcliffe, T Butterworth and B Proctor (eds), Fundamental Themes in Clinical Supervision. London: Routledge, 2001:25-46.

37. Graves C, Hanson D. The Multiple Mentoring Model of Student Supervision: A Fit for Contemporary Practice. https://www.duq.edu/assets/Documents/occupational-therapy/FieldworkEducation/Suggested\%20Readings/The\%20multiple\%20mentoring\%20model\%20of\%20student\%20supervision.pdf. Published May 12, 2014. Accessed May 14, 2020.

38. Jordon SE, Shearer EM. An exploration of supervision delivered via clinical video telehealth (CVT). Train Educ in Prof Psychol. 2019;13(4):323-330. http://dx.doi.org/10.1037/tep0000245. Published September 16, 2019. Accessed June 22, 2020. 
39. American Occupational Therapy Association. OT and Telehealth in the Age of COVID-19. https://www.aota.org/Practice/Manage/telehealth/coronavirus.aspx. Published 2020. Accessed June 22, 2020.

40. Allied Health Professions Council. Recommended Guidelines for Tele-Supervision of Conditionally Registered Occupational Therapists, Physiotherapists and Speech-Language Therapists during COVID-19 Circuit Breaker.

https://www.healthprofessionals.gov.sg/docs/librariesprovider5/forms-anddownloads/20200421_recommended_guidelines_on_tele-supervision.pdf?sfvrsn=6f69cbec_2. Published 2020. Accessed June 22, 2020.

41. Redpath AA, Gill SD, Finlay N, et al. Public sector physiotherapists believe that staff supervision should be broad ranging, individualized, structured, and based on needs and goals: A qualitative study. J Physiother. 2015;61(4):210-216. doi:10.1016/j.jphys.2015.08.002.

42. Physiotherapy Board of Australia. Supervision Guidelines for Physiotherapy. https://www.physiotherapyboard.gov.au/documents/default.aspx?record=WD10\%2F1394\&dbid=AP\&chksum=Dv\%2F9p8\% 2BmgiXfLUz7BUd5Uw\%3D\%3D. Published June 18, 2012. Accessed June 22, 2020.

43. Brough $P$, Timms $C$, O'Driscoll M, et al. Work-life balance: A longitudinal evaluation of a new measure across Australia and New Zealand workers. Int J Hum Resour Manag. 2014;25(19):2724-2744. doi:10.1080/09585192.2014.899262.

44. Manahan CM, Hardy CL, MacLeod MLP. Personal characteristics and experiences of long-term allied health professionals in rural and northern British Columbia. Rural Remote Health. 2009;9(4):1-13. doi:10.22605/RRH1238. 